\title{
Erratum: Analysis of a parametrically driven exchange-type gate and a two-photon excitation gate between superconducting qubits [Phys. Rev. A 96, 062323 (2017)]
}

Marco Roth, Marc Ganzhorn, Nikolaj Moll, Stefan Filipp, Gian Salis, and Sebastian Schmidt

(Received 13 April 2018; published 24 April 2018)

DOI: 10.1103/PhysRevA.97.049903

In the original paper there are typographical errors in Eqs. (14) and (16). The iswAP interaction is given by $\sigma_{1}^{x} \sigma_{2}^{x}+\sigma_{1}^{y} \sigma_{2}^{y}$ such that Eq. (14) should read

$$
H_{\mathrm{eff}} \approx \Omega_{\mathrm{eff}}^{-}\left(\sigma_{1}^{x} \sigma_{2}^{x}+\sigma_{1}^{y} \sigma_{2}^{y}\right) .
$$

Conversely, the bSWAP interaction is given by $\sigma_{1}^{x} \sigma_{2}^{x}-\sigma_{1}^{y} \sigma_{2}^{y}$ such that Eq. (16) should read

$$
H_{\text {eff }} \approx \Omega_{\text {eff }}^{+}\left(\sigma_{1}^{x} \sigma_{2}^{x}-\sigma_{1}^{y} \sigma_{2}^{y}\right) .
$$

Additionally, in the legend of Fig. 4, the blue dashed and solid lines represent the result of the bSWAP gate to first and second order.

Finally, the round brackets in Eq. (B2) should be square brackets as they denote a commutator. The correct form of Eq. (B2) is

$$
H_{\text {eff }}=H_{0}+[S, V]+\frac{1}{2}\left[S,\left[S, H_{0}\right]\right]+\frac{i}{2}\left[S, \frac{\partial S}{\partial t}\right] .
$$

The results are not altered by these typographical errors. 